Acta Crystallographica Section A

Foundations and

Advances

ISSN 2053-2733

\section{Seitz symbols for crystallographic symmetry operations}

\author{
A. Michael Glazer, ${ }^{a}$ Mois I. Aroyo ${ }^{\mathrm{b} *}$ and André Authier ${ }^{\mathrm{c}}$ \\ ${ }^{\mathrm{a}}$ Department of Physics, University of Oxford, Parks Road, Oxford, United Kingdom, ${ }^{\mathbf{b}}$ Departamento de Física de \\ la Materia Condensada, Universidad del País Vasco (UPV/EHU), Bilbao, Spain, and Institut de Minéralogie, de \\ Physique des Matériaux, et de Cosmochimie, UPMC, Paris, France. Correspondence e-mail: mois.aroyo@ehu.es
}

\begin{abstract}
The aim of this report is to describe the Seitz notation for symmetry operations adopted by the Commission on Crystallographic Nomenclature as the standard convention for Seitz symbolism of the International Union of Crystallography. The established notation follows the existing crystallographic conventions in the descriptions of symmetry operations.
\end{abstract}

\section{Introduction}

Apart from the notation used in International Tables for Crystallography, Volume A (referred to as ITA) for the geometric interpretation of the matrix-column representation of symmetry operations $(\boldsymbol{W}, \boldsymbol{w})$, there is another notation which has been adopted and is widely used by solid-state physicists and chemists. This is the so-called Seitz notation $\{\boldsymbol{R} \mid \boldsymbol{v}\}$ introduced by Seitz in a series of papers on the matrix-algebraic development of crystallographic groups (Seitz, 1935).

During the 22nd Congress and General Assembly of the International Union of Crystallography (IUCr) in Madrid (2011), it was decided that the Commission on Crystallographic Nomenclature should try to establish a convention for Seitz notation that closely follows the already accepted crystallographic conventions for the description of symmetry operations. It was agreed that Seitz notation should not appear in the printed volume of ITA, but could be added to the online versions of International Tables for Crystallography.

The aim of the present contribution is to introduce the Seitz notation for symmetry operations adopted by the Commission on Crystallographic Nomenclature as the standard IUCr convention for Seitz symbolism.

\section{Description of the standard Seitz notation}

Seitz symbols $\{\boldsymbol{R} \mid \boldsymbol{v}\}$ are essentially shorthand descriptions of the matrix-column representations of the symmetry operations of the space groups. They consist of two parts: a rotation (or linear) part $\boldsymbol{R}$ and a translation part $\boldsymbol{v}$. The Seitz symbol is specified between braces and the rotational and the translational parts are separated by a vertical line. The translation parts $\boldsymbol{v}$ correspond exactly to the translation parts of the coordinate triplets $\boldsymbol{w}$ of the General position blocks of the space-group tables of ITA. The rotation parts $\boldsymbol{R}$ consist of symbols that specify (i) the type and the order of the symmetry operation, and (ii) the orientation of the corresponding symmetry element with respect to the basis. The orientation is denoted by the direction of the axis for rotations or rotoinversions, or the direction of the normal to reflection planes. (Note that in the latter case this is in contrast to the way the orientation of reflection planes is given in the Symmetry operations block of ITA.)

There are many different ways in which one finds the linear parts of Seitz symbols denoted in the literature (Litvin \& Kopsky, 2011).
According to the conventions approved by the Commission on Crystallographic Nomenclature, the symbol $\boldsymbol{R}$ is 1 and $\overline{1}$ for the identity and the inversion, respectively, and $m$ for reflections; the symbols $2,3,4$ and 6 are used for rotations and $\overline{3}, \overline{4}$ and $\overline{6}$ are used for rotoinversions. For rotations and rotoinversions of order higher than 2 , a superscript + or - is used to indicate the sense of the rotation. Subscripts of the symbols $\boldsymbol{R}$ denote the characteristic direction of the operation: for example, the subscripts 100,010 and $1 \overline{1} 0$ refer to the directions [100], [010] and [110], respectively.

Examples:

(a) Consider the coordinate triplets of the general positions of $P 2{ }_{1} 22$ (No. 18):
(1) $x, y, z$
(2) $\bar{x}, \bar{y}, z$
(3) $\bar{x}+\frac{1}{2}, y+\frac{1}{2}, \bar{z}$
(4) $x+\frac{1}{2}, \bar{y}+\frac{1}{2}, \bar{z}$

The corresponding geometric interpretations of symmetry operations in ITA are given by
(1) 1
(2) $20,0, z$
(3) $2\left(0, \frac{1}{2}, 0\right) \frac{1}{4}, y, 0$
(4) $2\left(\frac{1}{2}, 0,0\right) x, \frac{1}{4}, 0$

In Seitz notation the symmetry operations are denoted by
(1) $\{1 \mid 0\}$
(2) $\left\{2_{001} \mid 0\right\}$
(3) $\left\{2_{010} \mid \frac{1}{2}, \frac{1}{2}, 0\right\}$
(4) $\left\{2_{100} \mid \frac{1}{2}, \frac{1}{2}, 0\right\}$

(b) Similarly, the general positions of $P 12_{1} / c 1$ (cell choice 1) (No. 14) are given as
(1) $x, y, z$
(2) $\bar{x}, y+\frac{1}{2}, \bar{z}+\frac{1}{2}$
(3) $\bar{x}, \bar{y}, \bar{z}$
(4) $x, \bar{y}+\frac{1}{2}, z+\frac{1}{2}$

The geometric descriptions given in the Symmetry operations block in ITA are

(1) 1

(2) $2\left(0, \frac{1}{2}, 0\right) 0, y, \frac{1}{4}$

(3) $\overline{1} 0,0,0$

(4) $c x, \frac{1}{4}, z$

which in Seitz notation are given as
(1) $\{1 \mid 0\}$
(2) $\left\{2_{010} \mid 0, \frac{1}{2}, \frac{1}{2}\right\}$
(3) $\{\overline{1} \mid 0\}$
(4) $\left\{m_{010} \mid 0, \frac{1}{2}, \frac{1}{2}\right\}$

The linear parts $\boldsymbol{R}$ of the Seitz symbols of the space-group symmetry operations are shown in Tables $1-3$. Each symbol $\boldsymbol{R}$ is specified by the shorthand notation of its $(3 \times 3)$ matrix representation (also known as the Jones' faithful representation symbol, cf. Bradley \& Cracknell, 1972), the type of symmetry operation and its orientation as described in the corresponding Symmetry operations block of the space-group tables of ITA. The sequence of $\boldsymbol{R}$ symbols in Table 1 corresponds to the numbering scheme of the general-position coordinate triplets of the space groups of the $m \overline{3} m$ crystal class, while those of Table 2 and Table 3 correspond to the general-position 
Table 1

Linear parts $\boldsymbol{R}$ of the Seitz symbols $\{\boldsymbol{R} \mid \boldsymbol{v}\}$ for space-group symmetry operations of cubic, tetragonal, orthorhombic, monoclinic and triclinic crystal systems.

Each symmetry operation is specified by the shorthand description of the rotation part of its matrix-column presentation, the type of symmetry operation and its characteristic direction.

\begin{tabular}{|c|c|c|c|c|}
\hline \multicolumn{4}{|c|}{ ITA description } & \multirow{2}{*}{$\begin{array}{l}\text { Seitz } \\
\text { symbol }\end{array}$} \\
\hline No. & Coordinate triplet & Type & Orientation & \\
\hline 1 & $x, y, z$ & 1 & & 1 \\
\hline 2 & $\bar{x}, \bar{y}, z$ & 2 & $0,0, z$ & $2_{001}$ \\
\hline 3 & $\bar{x}, y, \bar{z}$ & 2 & $0, y, 0$ & $2_{010}$ \\
\hline 4 & $x, \bar{y}, \bar{z}$ & 2 & $x, 0,0$ & $2_{100}$ \\
\hline 5 & $z, x, y$ & $3^{+}$ & $x, x, x$ & $3_{111}^{+}$ \\
\hline 6 & $z, \bar{x}, \bar{y}$ & $3^{+}$ & $\bar{x}, x, \bar{x}$ & $3_{\overline{1} 1 \overline{1}}^{+}$ \\
\hline 7 & $\bar{z}, \bar{x}, y$ & $3^{+}$ & $x, \bar{x}, \bar{x}$ & $3_{1 \overline{1} \overline{1}}^{+}$ \\
\hline 8 & $\bar{z}, x, \bar{y}$ & $3^{+}$ & $\bar{x}, \bar{x}, x$ & $3_{\overline{1} 1}^{+}$ \\
\hline 9 & $y, z, x$ & $3^{-}$ & $x, x, x$ & $3_{111}^{-}$ \\
\hline 10 & $\bar{y}, z, \bar{x}$ & $3^{-}$ & $x, \bar{x}, \bar{x}$ & $3_{1 \overline{1} \overline{1}}^{-}$ \\
\hline 11 & $y, \bar{z}, \bar{x}$ & $3^{-}$ & $\bar{x}, \bar{x}, x$ & $3_{\overline{1} \overline{1} 1}^{-}$ \\
\hline 12 & $\bar{y}, \bar{z}, x$ & $3^{-}$ & $\bar{x}, x, \bar{x}$ & $3_{\overline{1} 1 \overline{1}}^{-}$ \\
\hline 13 & $y, x, \bar{z}$ & 2 & $x, x, 0$ & $2_{110}$ \\
\hline 14 & $\bar{y}, \bar{x}, \bar{z}$ & 2 & $x, \bar{x}, 0$ & $2_{1 \overline{1} 0}$ \\
\hline 15 & $y, \bar{x}, z$ & $4^{-}$ & $0,0, z$ & $4_{001}^{-}$ \\
\hline 16 & $\bar{y}, x, z$ & $4^{+}$ & $0,0, z$ & $4_{001}^{+}$ \\
\hline 17 & $x, z, \bar{y}$ & $4^{-}$ & $x, 0,0$ & $4_{100}^{-}$ \\
\hline 18 & $\bar{x}, z, y$ & 2 & $0, y, y$ & $2_{011}$ \\
\hline 19 & $\bar{x}, \bar{z}, \bar{y}$ & 2 & $0, y, \bar{y}$ & $2_{01 \overline{1}}$ \\
\hline 20 & $x, \bar{z}, y$ & $4^{+}$ & $x, 0,0$ & $4_{100}^{+}$ \\
\hline 21 & $z, y, \bar{x}$ & $4^{+}$ & $0, y, 0$ & $4_{010}^{+}$ \\
\hline 22 & $z, \bar{y}, x$ & 2 & $x, 0, x$ & $2_{101}$ \\
\hline 23 & $\bar{z}, y, x$ & $4^{-}$ & $0, y, 0$ & $4_{010}^{-}$ \\
\hline 24 & $\bar{z}, \bar{y}, \bar{x}$ & 2 & $\bar{x}, 0, x$ & $2_{\overline{1} 01}$ \\
\hline 25 & $\bar{x}, \bar{y}, \bar{z}$ & $\overline{1}$ & & $\overline{1}$ \\
\hline 26 & $x, y, \bar{z}$ & $m$ & $x, y, 0$ & $m_{001}$ \\
\hline 27 & $x, \bar{y}, z$ & $m$ & $x, 0, z$ & $m_{010}$ \\
\hline 28 & $\bar{x}, y, z$ & $m$ & $0, y, z$ & $m_{100}$ \\
\hline 29 & $\bar{z}, \bar{x}, \bar{y}$ & $\overline{3}^{+}$ & $x, x, x$ & $\overline{3}_{111}^{+}$ \\
\hline 30 & $\bar{z}, x, y$ & $\overline{3}^{+}$ & $\bar{x}, x, \bar{x}$ & $\overline{3}_{\overline{1} 1 \overline{1}}^{+}$ \\
\hline 31 & $z, x, \bar{y}$ & $\overline{3}^{+}$ & $x, \bar{x}, \bar{x}$ & $\overline{3}_{1 \overline{1} \overline{1}}^{+}$ \\
\hline 32 & $z, \bar{x}, y$ & $\overline{3}^{+}$ & $\bar{x}, \bar{x}, x$ & $3_{\overline{11} 1}^{+}$ \\
\hline 33 & $\bar{y}, \bar{z}, \bar{x}$ & $\overline{3}^{-}$ & $x, x, x$ & $\overline{3}_{111}^{-}$ \\
\hline 34 & $y, \bar{z}, x$ & $\overline{3}^{-}$ & $x, \bar{x}, \bar{x}$ & $\overline{3}_{1 \overline{1} \overline{1}}^{-}$ \\
\hline 35 & $\bar{y}, z, x$ & $\overline{3}^{-}$ & $\bar{x}, \bar{x}, x$ & $\overline{3}_{\overline{1} \overline{1} 1}$ \\
\hline 36 & $y, z, \bar{x}$ & $\overline{3}^{-}$ & $\bar{x}, x, \bar{x}$ & $\overline{3}_{\overline{1} 1 \overline{1}}^{-}$ \\
\hline 37 & $\bar{y}, \bar{x}, z$ & $m$ & $x, \bar{x}, z$ & $m_{110}$ \\
\hline 38 & $y, x, z$ & $m$ & $x, x, z$ & $m_{1 \overline{1} 0}$ \\
\hline 39 & $\bar{y}, x, \bar{z}$ & $\overline{4}^{-}$ & $0,0, z$ & $\overline{4}_{001}^{-}$ \\
\hline 40 & $y, \bar{x}, \bar{z}$ & $\overline{4}^{+}$ & $0,0, z$ & $\overline{\mathbf{4}}_{001}^{+}$ \\
\hline 41 & $\bar{x}, \bar{z}, y$ & $\overline{4}^{-}$ & $x, 0,0$ & $\overline{4}_{100}^{-}$ \\
\hline 42 & $x, \bar{z}, \bar{y}$ & $m$ & $x, y, \bar{y}$ & $m_{011}$ \\
\hline 43 & $x, z, y$ & $m$ & $x, y, y$ & $m_{01 \overline{1}}$ \\
\hline 44 & $\bar{x}, z, \bar{y}$ & $\overline{4}^{+}$ & $x, 0,0$ & $\overline{4}_{100}^{+}$ \\
\hline 45 & $\bar{z}, \bar{y}, x$ & $\overline{4}^{+}$ & $0, y, 0$ & $\overline{4}_{010}^{+}$ \\
\hline 46 & $\bar{z}, y, \bar{x}$ & $m$ & $\bar{x}, y, x$ & $m_{101}$ \\
\hline 47 & $z, \bar{y}, \bar{x}$ & $\overline{4}^{-}$ & $0, y, 0$ & $\overline{4}_{010}^{-}$ \\
\hline 48 & $z, y, x$ & $m$ & $x, y, x$ & $m_{\overline{1} 01}$ \\
\hline
\end{tabular}

Table 2

Linear parts $\boldsymbol{R}$ of the Seitz symbols $\{\boldsymbol{R} \mid \boldsymbol{v}\}$ for space-group symmetry operations of hexagonal and trigonal crystal systems.

Each symmetry operation is specified by the shorthand description of the rotation part of its matrix-column presentation, the type of symmetry operation and its characteristic direction.

\begin{tabular}{|c|c|c|c|c|}
\hline \multicolumn{4}{|c|}{ ITA description } & \multirow{2}{*}{$\begin{array}{l}\text { Seitz } \\
\text { symbol }\end{array}$} \\
\hline No. & Coordinate triplet & Type & Orientation & \\
\hline 1 & $x, y, z$ & 1 & & 1 \\
\hline 2 & $\bar{y}, x-y, z$ & $3^{+}$ & $0,0, z$ & $3_{001}^{+}$ \\
\hline 3 & $\bar{x}+y, \bar{x}, z$ & $3^{-}$ & $0,0, z$ & $3_{001}^{-}$ \\
\hline 4 & $\bar{x}, \bar{y}, z$ & 2 & $0,0, z$ & $2_{001}$ \\
\hline 5 & $y, \bar{x}+y, z$ & $6^{-}$ & $0,0, z$ & $6_{001}^{-}$ \\
\hline 6 & $x-y, x, z$ & $6^{+}$ & $0,0, z$ & $6_{001}^{+}$ \\
\hline 7 & $y, x, \bar{z}$ & 2 & $x, x, 0$ & $2_{110}$ \\
\hline 8 & $x-y, \bar{y}, \bar{z}$ & 2 & $x, 0,0$ & $2_{100}$ \\
\hline 9 & $\bar{x}, \bar{x}+y, \bar{z}$ & 2 & $0, y, 0$ & $2_{010}$ \\
\hline 10 & $\bar{y}, \bar{x}, \bar{z}$ & 2 & $x, \bar{x}, 0$ & $2_{1 \overline{1} 0}$ \\
\hline 11 & $\bar{x}+y, y, \bar{z}$ & 2 & $x, 2 x, 0$ & $2_{120}$ \\
\hline 12 & $x, x-y, \bar{z}$ & 2 & $2 x, x, 0$ & $2_{210}$ \\
\hline 13 & $\bar{x}, \bar{y}, \bar{z}$ & $\overline{1}$ & & $\overline{1}$ \\
\hline 14 & $y, \bar{x}+y, \bar{z}$ & $\overline{3}^{+}$ & $0,0, z$ & $\overline{3}_{001}^{+}$ \\
\hline 15 & $x-y, x, \bar{z}$ & $\overline{3}^{-}$ & $0,0, z$ & $\overline{3}_{001}^{-}$ \\
\hline 16 & $x, y, \bar{z}$ & $m$ & $x, y, 0$ & $m_{001}$ \\
\hline 17 & $\bar{y}, x-y, \bar{z}$ & $\overline{6}^{-}$ & $0,0, z$ & $\bar{\sigma}_{001}^{-}$ \\
\hline 18 & $\bar{x}+y, \bar{x}, \bar{z}$ & $\overline{6}^{+}$ & $0,0, z$ & $\bar{\sigma}_{001}^{+}$ \\
\hline 19 & $\bar{y}, \bar{x}, z$ & $m$ & $x, \bar{x}, z$ & $m_{110}$ \\
\hline 20 & $\bar{x}+y, y, z$ & $m$ & $x, 2 x, z$ & $m_{100}$ \\
\hline 21 & $x, x-y, z$ & $m$ & $2 x, x, z$ & $m_{010}$ \\
\hline 22 & $y, x, z$ & $m$ & $x, x, z$ & $m_{1 \overline{1} 0}$ \\
\hline 23 & $x-y, \bar{y}, z$ & $m$ & $x, 0, z$ & $m_{120}$ \\
\hline 24 & $\bar{x}, \bar{x}+y, z$ & $m$ & $0, y, z$ & $m_{210}$ \\
\hline
\end{tabular}

Table 3

Linear parts $\boldsymbol{R}$ of the Seitz symbols $\{\boldsymbol{R} \mid \boldsymbol{v}\}$ for symmetry operations of rhombohedral space groups (rhombohedral-axes setting).

Each symmetry operation is specified by the shorthand description of the rotation part of its matrix-column presentation, the type of symmetry operation and its characteristic direction.

\begin{tabular}{rllll}
\hline \multicolumn{2}{l}{ ITA description } & & & $\begin{array}{l}\text { Seitz } \\
\text { symbol }\end{array}$ \\
\cline { 1 - 2 } No. & Coordinate triplet & Type & Orientation & 1 \\
\hline 1 & $x, y, z$ & 1 & & $3_{111}^{+}$ \\
2 & $z, x, y$ & $3^{+}$ & $x, x, x$ & $3_{111}^{-}$ \\
3 & $y, z, x$ & $3^{-}$ & $x, x, x$ & $2_{\overline{1} 01}$ \\
4 & $\bar{z}, \bar{y}, \bar{x}$ & 2 & $\bar{x}, 0, x$ & $2_{1 \overline{1} 0}$ \\
5 & $\bar{y}, \bar{x}, \bar{z}$ & 2 & $x, \bar{x}, 0$ & $2_{01 \overline{1}}$ \\
6 & $\bar{x}, \bar{z}, \bar{y}$ & 2 & $0, y, \bar{y}$ & $\overline{1}^{-}$ \\
7 & $\bar{x}, \bar{y}, \bar{z}$ & $\overline{1}$ & & $\overline{3}_{111}^{+}$ \\
8 & $\bar{z}, \bar{x}, \bar{y}$ & $\overline{3}^{+}$ & $x, x, x$ & $\overline{3}_{111}^{-}$ \\
9 & $\bar{y}, \bar{z}, \bar{x}$ & $\overline{3}^{-}$ & $x, x, x$ & $m_{\overline{101}}$ \\
10 & $z, y, x$ & $m$ & $x, y, x$ & $m_{1 \overline{1} 0}$ \\
11 & $y, x, z$ & $m$ & $x, x, z$ & $m_{01 \overline{1}}$ \\
12 & $x, z, y$ & $m$ & $x, y, y$ & \\
\hline
\end{tabular}


sequences of the space groups of the $6 / \mathrm{mmm}$ and $\overline{3} \mathrm{~m}$ (rhombohedral axes) crystal classes.

The same symbols $\boldsymbol{R}$ can be used for the construction of Seitz symbols for the symmetry operations of subperiodic groups or magnetic groups, or for the designation of the symmetry operations of the point groups of space groups. [One should note that the Seitz symbols given in International Tables for Crystallography, Vol. E (2010) and the IUCr e-book on magnetic groups (Litvin, 2013) differ from the standard symbols adopted by the Commission on Crystallographic Nomenclature.]

From the description given above, it is clear that Seitz symbols can be considered as shorthand modifications of the matrix-column presentation $(\boldsymbol{W}, \boldsymbol{w})$ of symmetry operations: the translation parts of $\{\boldsymbol{R} \mid \boldsymbol{v}\}$ and $(\boldsymbol{W}, \boldsymbol{w})$ coincide, while the different $(3 \times 3)$ matrices $\boldsymbol{W}$ are represented by the symbols $\boldsymbol{R}$ shown in Tables $1-3$. As a result, the expressions for the product and the inverse of symmetry operations in Seitz notation are rather similar to those of the matrix-column pairs $(\boldsymbol{W}, \boldsymbol{w})$ :

(i) Product of symmetry operations: $\left\{\boldsymbol{R}_{1} \mid \boldsymbol{v}_{1}\right\}\left\{\boldsymbol{R}_{2} \mid \boldsymbol{v}_{2}\right\}=$ $\left\{\boldsymbol{R}_{1} \boldsymbol{R}_{2} \mid \boldsymbol{R}_{1} \boldsymbol{v}_{2}+\boldsymbol{v}_{1}\right\}$

(ii) Inverse of a symmetry operation: $\{\boldsymbol{R} \mid \boldsymbol{v}\}^{-1}=\left\{\boldsymbol{R}^{-1} \mid-\boldsymbol{R}^{-1} \boldsymbol{v}\right\}$.

Similarly, the action of a symmetry operation $\{\boldsymbol{R} \mid \boldsymbol{v}\}$ on a point $\boldsymbol{x}$ is given by $\{\boldsymbol{R} \mid \boldsymbol{v}\} \boldsymbol{x}=\boldsymbol{R} \boldsymbol{x}+\boldsymbol{v}$.

The rotation parts of the Seitz symbols partly resemble the geometric description symbols of symmetry operations listed in the Symmetry operations blocks of the space-group tables of ITA: $\boldsymbol{R}$ contain the information on the type and order of the symmetry operation, and its characteristic direction. The Seitz symbols do not directly indicate the location of the symmetry operation, or its glide or screw component, if any.

\section{Availability of the standard Seitz symbols}

The standard Seitz symbols can be computer generated and the corresponding programs have already been developed and implemented in the Bilbao Crystallographic Server (http://www.cryst. ehu.es): the computer access tool GENPOS shows the corresponding Seitz symbols for the symmetry operations of space, layer and rod groups.

\section{Conclusions}

The Seitz notation of the space-group symmetry operations adopted by the Commission on Crystallographic Nomenclature as the standard IUCr convention for Seitz symbolism is described in this report. It is recommended that the Seitz symbols are included in the online version of the space-group tables of ITA and that the adopted notation is applied in the description of symmetry operations in subperiodic space groups, magnetic groups or for the designation of the symmetry operations of the crystallographic point groups.

An interesting discussion on the symbol for designation of reflections took place among the members of the Commission on Crystallographic Nomenclature during the vote on Seitz notation. There was a proposal to use the symbol $\overline{2}$ instead of $m$ with the argument that the symbol $\overline{2}$ is more consistent from a mathematical point of view and adds clarity to the relationships between rotations and rotoinversions. Although in general the arguments in favour of the $\overline{2}$ symbol were accepted, the vast majority of commission members did not support the proposal because of the intuitiveness of the symbol $m$, its popularity and its long tradition of usage by the crystallographic community. Nevertheless, because of the pedagogical benefits of the symbol $\overline{2}$, it is recommended that the definition of $m$ as $\overline{2}$ is emphasized whenever the opportunity arises.

The authors would like to thank all the members of the Commission on Crystallographic Nomenclature, past and present, who took part in the fruitful discussions on Seitz symbolism.

\section{References}

Bradley, C. J. \& Cracknell, A. P. (1972). The Mathematical Theory of Symmetry in Solids: Representation Theory for Point Groups and Space Groups. Oxford: Clarendon Press.

International Tables for Crystallography (1983). Volume A, Space-Group Symmetry, edited by Th. Hahn. Dordrecht: Kluwer Academic Publishers. (Abbreviated ITA, fifth revised edition 2002).

International Tables for Crystallography (2010). Volume E, Subperiodic Groups, edited by V. Kopský and D. B. Litvin, 2nd ed. Chichester: Wiley.

Litvin, D. B. (2013). Magnetic Group Tables. IUCr e-book. http://www.iucr.org/ publ/978-0-9553602-2-0.

Litvin, D. B. \& Kopský, V. (2011). Acta Cryst. A67, 415-418.

Seitz, F. (1935). Z. Kristallogr. 91, 336-366. 